\title{
A new chaotic system with line of equilibria: dynamics, passive control and circuit design
}

\author{
Aceng Sambas', Mustafa Mamat' ${ }^{2}$, Ayman Ali Arafa ${ }^{3}$ Gamal M Mahmoud $^{4}$, \\ Mohamad Afendee Mohamed ${ }^{5}$, W. S. Mada Sanjaya ${ }^{6}$ \\ ${ }^{1}$ Department of Mechanical Engineering, Universitas Muhammadiyah Tasikmalaya, Indonesia \\ ${ }^{2,5}$ Faculty of Informatics and Computing, Universiti Sultan Zainal Abidin, Kuala Terengganu, Malaysia \\ ${ }^{3}$ Department of Mathematics, Faculty of Science, Sohag University, Egypt \\ ${ }^{4}$ Department of Mathematics, Faculty of Science, Assiut University, Egypt \\ ${ }^{6}$ Department of Physics, Universitas Islam Negeri Sunan Gunung Djati Bandung, Indonesia
}

\section{Article Info}

Article history:

Received Jan 9, 2018

Revised Mar 7, 2019

Accepted Mar 11, 2019

\section{Keywords:}

Chaotic system

Dynamical system

Electronic circuit

Line equilibrium

Passive control

\begin{abstract}
A new chaotic system with line equilibrium is introduced in this paper. This system consists of five terms with two transcendental nonlinearities and two quadratic nonlinearities. Various tools of dynamical system such as phase portraits, Lyapunov exponents, Kaplan-Yorke dimension, bifurcation diagram and Poincarè map are used. It is interesting that this system has a line of fixed points and can display chaotic attractors. Next, this paper discusses control using passive control method. One example is given to insure the theoretical analysis. Finally, for the new chaotic system, an electronic circuit for realizing the chaotic system has been implemented. The numerical simulation by using MATLAB 2010 and implementation of circuit simulations by using MultiSIM 10.0 have been performed in this study.
\end{abstract}

Copyright (ㅇ 2019 Institute of Advanced Engineering and Science. All rights reserved.

\section{Corresponding Author:}

Aceng Sambas,

Department of Mechanical Engineering,

Universitas Muhammadiyah Tasikmalaya,

Tamansari Gobras Street Km 2.5, Tasikmalaya, West Java, Indonesia.

Email: acengs@umtas.ac.id

\section{INTRODUCTION}

Discovering chaotic attractor is an important issue in chaotic systems. We can classify two kinds of chaotic attractors: self-excited attractors and hidden attractors [1-2]. The chaotic system such as Lorenz

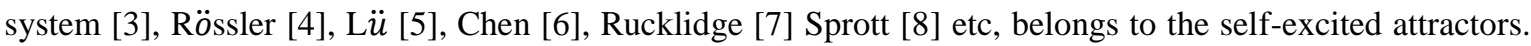
The chaotic systems with hidden attractors are divided into three parts: (a) system with no equilibria [9] (b) system with stable equilibria [10] and (c) system with infinite number of equilibria [11]. Hidden attractors have been used in applied models such as a model of the phase-locked loop (PLL) [12], aircraft flight control systems [13], drilling system actuated by induction motor [14], Lorenz-like system describing convective fluid motion in rotating cavity [15] and a multilevel DC/DC converter [16].

Motivated by the major work of Jafari and Sprott, researchers focused on chaotic systems with line of equilibria. The nine simple chaotic flows with line of equilibria were proposed by Jafari and Sprott [17]. Five novel chaotic system with a line of equilibria and two parallel lines were proposed by Li and Sprott [18]. Li and Sprott have presented chaotic systems with a line of equilibria and two perpendicular lines of equilibria by using signum functions and absolute-value functions [19]. In addition, Li et al reported a hyperchaotic system with an infinite number of equilibria and circuit design [20]. Hyperchaos and horseshoe in a $4 \mathrm{D}$ memristive system with a line of equilibria were proposed in [21]. The simplest 4-D chaotic system 
with line of equilibria, by applying a state feed-back control to Rössler equation was represented by Singh and Roy [22].

Various control methods have been used for controlling chaotic systems such as adaptive control [23], sliding mode control [24], active control [25], passive control [26], Pecora-Carroll control [27], impulsive control [28], fuzzy control [29], optimal control [30], digital redesign control [31] and many others [32-33]. The concept of passivity theory has been found to be a nice tool in in various domains of science and engineering such as robotic [34], signal processing [35], permanent-magnet synchronous motors chaotic system [36], hopfield neural network [37], nuclear spin generator system [38], synchronization [39], compass-like biped robot [40] and complexity [41].

This paper introduces a new chaotic system with line of equilibria. Our new chaotic system has five terms with two transcendental nonlinearities and two quadratic nonlinearities. Basic properties of the new chaotic system are analyzed, including such as equilibrium points, Lyapunov exponents, Kaplan-Yorke dimension, bifurcation diagram and Poincaré map. Finally, passive control and circuit design.

The rest of our paper is organized as follows. In Section 2, description of the new chaotic system with line of equilibria is presented. In addition, the dynamics properties of the new chaotic system are investigated. Stability analysis of the system is presented in Section 3. Some definitions and theorems of passive control are presented in Section 4 and control of this chaotic system using passive control is described in section 5. In Section 6, a new electronic implementation of the new chaotic system is described and examined in MultiSIM. Finally, conclusions are drawn in Section 7.

\section{DYNAMICAL ANALYSIS OF A NEW CHAOTIC SYSTEM WITH LINE OF EQUILIBRIA} system as:

In this part, inspired by the method and structure proposed in [19], we present a new chaotic

$$
\left\{\begin{array}{l}
\dot{x}=y z \\
\dot{y}=x|x|-y|y| \\
\dot{z}=a|x|-b y^{2}
\end{array}\right.
$$

where $x, y, z$ are state variables $a$ and $b$ are positive system parameters. Here the parameter $a$ is a control parameter to control the amplitude and frequency of all variables.

The new chaotic system (1) exhibits chaotic behavior as shown in Figure 1

$$
a=1.6, b=0.8
$$

and with the initial conditions

$$
x(0)=0.2, y(0)=0.2, z(0)=0.2
$$

The fourth order Runge-Kutta method is used for employing the numerical simulations. Moreover the Lyapunov exponents of the new system (1) are calculated using Wolf algorithm [42].

$$
L E_{1}=0.11026, L E_{2}=0, L E_{3}=-1.66103
$$

As seen in Figure 2 (a). A positive Lyapunov exponent reveals the presence of chaotic system. Simulation is run for 50.000 seconds.

The Kaplan-Yorke dimension of the new chaotic system (1) is calculated as

$$
\mathrm{D}_{\mathrm{KY}}=2+\frac{L E_{1}+L E_{2}}{\left|L E_{3}\right|}=2.066
$$

Therefore, system (1) is really chaotic with fractional dimension.

a. Fix $a=1.6$ and vary $b$ :

For this case $b=0.46$, the Lyapunov exponents for system (1) are: $L E_{1}=0.0230, L E_{2}=-0.0169$, $L E_{3}=-2.4458$. It is clear that (1) has chaotic attractors for $b \geq 0.46$. For $b=0.45$, the Lyapunov exponents for system (1) are: $L E_{1}=0.0223, L E_{2}=-0.0284, L E_{3}=-2.4847$. Thus, periodic behavior can be seen in the system for $b<0.46$. 
b. Fix $b=0.8$ and vary $a$

For $a=0.1$, the Lyapunov exponents for system (1) are: $L E_{1}=0.0063, L E_{2}=-0.0053, L E_{3}=-0.1072$. It is clear that (1) has chaotic attractors for $a \geq 0.1$ and for $a=0.09$, the Lyapunov exponents for system (1) are: $L E_{1}=0.0048, L E_{2}=-0.0078, L E_{3}=-0.1048$. Thus, periodic behavior can be seen in the system for $a<0.09$.

The bifurcation diagram and Lyapunov exponent spectrum of new chaotic system (1) for a=1.6, $\mathrm{b}=0.8$ and initial conditions $\mathrm{x}(0)=0.2, \mathrm{y}(0)=0.2, \mathrm{z}(0)=0.2$ are plotted in Figures 2(b) and 2(c), respectively. As shown in Figures 2(b) and 2(c), the system (1) has periodic behavior or chaotic behavior by varying the value of the parameter $b$. When the value of $b<0.46$, system (1) exhibits periodic state and when $\mathrm{b} \geq 0.46$, the system (1) shows complex behavior. In addition, the Poincare map of the system (1) in Figure 2(d) also reflects properties of chaos.

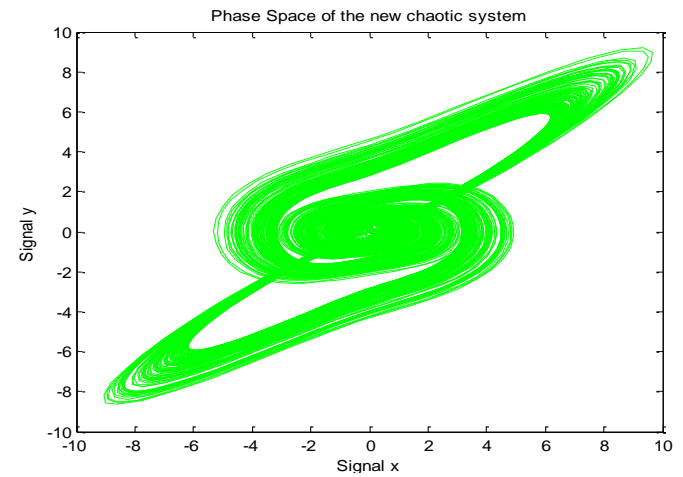

(a)

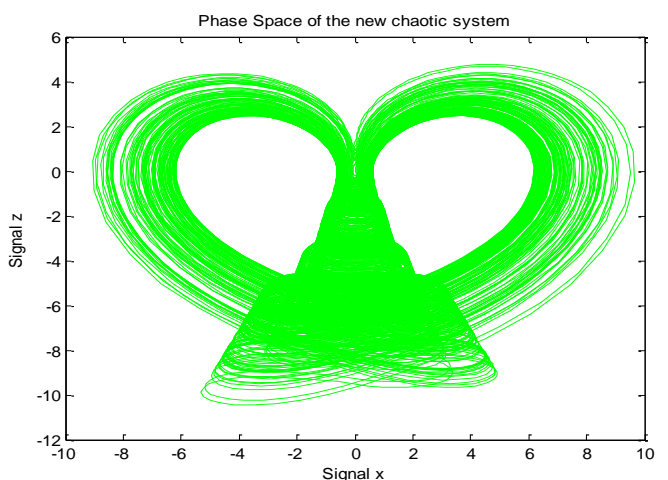

(c)

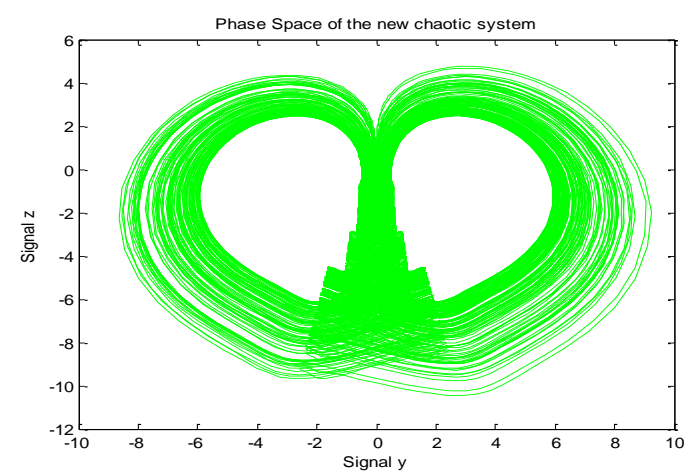

(b)

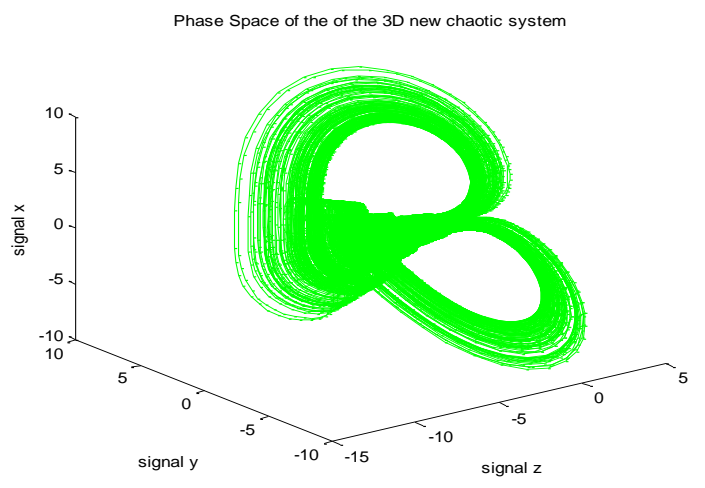

(d)

Figure 1. Numerical simulation results using MATLAB, for $a=1.6$ and $b=0.8$, in (a) $x-y$ plane, (b) $y-z$ plane, (c) $x-z$ plane and (d) $x-y-z$ plane

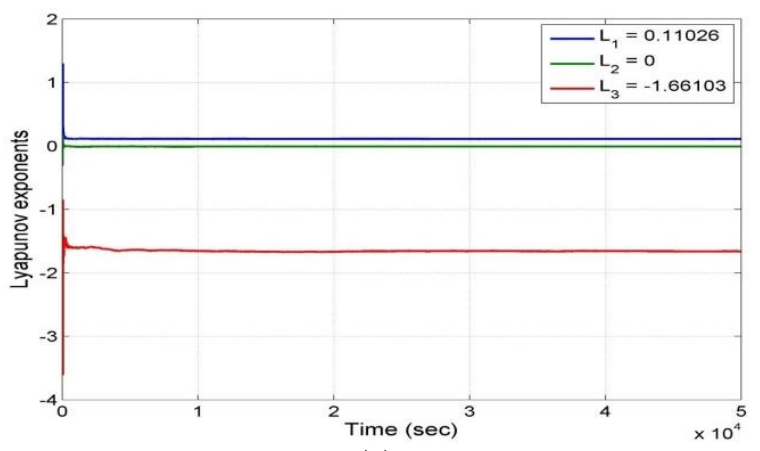

(a)

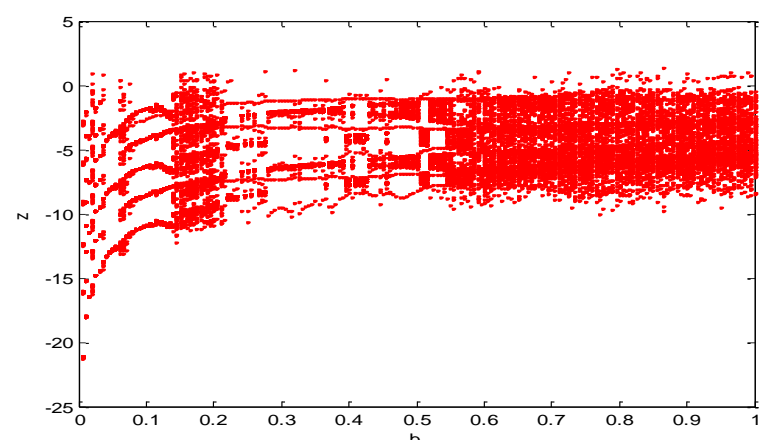

(b)

Figure 2 Complex analysis of new chaotic system (1) using MATLAB for $a=1.6$

(a) The Lyapunov exponents of the new system (1) (b) Bifurcation diagram of the new chaotic system (1) with $b$ as varying parameter 


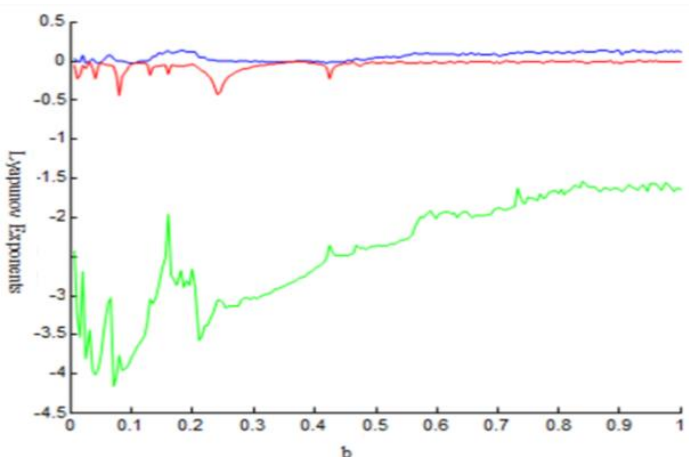

(c)

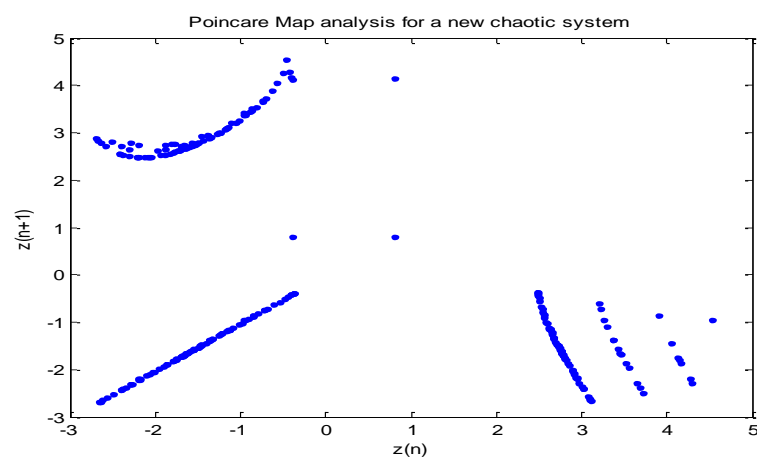

(d)

Figure 2 Complex analysis of new chaotic system (1) using MATLAB for $a=1.6$

(c) Lyapunov spectrum of system (1) when varying the parameter $b$ (d) Poincare map of system (1)

\section{EQUILIBRIUM AND STABILITY}

The equilibrium points of the new chaotic system (1) are obtained by solving the following system.

$$
\left\{\begin{array}{l}
0=y z \\
0=x|x|-y|y| \\
0=a|x|-b y^{2}
\end{array}\right.
$$

System (1) has a line equilibrium $E_{z}=(0,0, z)$ and four nontrivial fixed points which are $\left( \pm \frac{a}{b}, \pm \frac{a}{b}, 0\right)$ and $\left( \pm \frac{a}{b}, \mp \frac{a}{b}, 0\right)$. The Jacobian matrix of the system (1) is given by

$$
J=\left[\begin{array}{ccc}
0 & z & y \\
|x|+x * \operatorname{sign}(x) & -(|y|+y * \operatorname{sign}(y)) & 0 \\
a * \operatorname{sign}(x) & -2 * b * y & 0
\end{array}\right]
$$

The characteristic equation can be written as

$$
\begin{gathered}
\lambda^{3}+(|x|+x * \operatorname{sign}(\mathrm{x})) \lambda^{2}+(-z|x|-(a y+x z) * \operatorname{sign}(\mathrm{x})) \lambda+ \\
2 b y^{2}|x|-a x y+\left(2 b x y^{2}-a y|x|\right) \operatorname{sign}(\mathrm{x})=0
\end{gathered}
$$

It is clear that the eigenvalues for system (1) at the line equilibrium $E_{z}$ are $\lambda_{1}=\lambda_{2}=\lambda_{3}=0$.

System (1) has four equilibrium points at $\mathrm{E}_{1,2}( \pm 2, \pm 2,0), \mathrm{E}_{3,4}(\mp 2, \mp 2,0)$ with $\mathrm{a}=1.6$ and $\mathrm{b}=0.8$. The eigenvalues at $E_{1}$ are

$$
\lambda_{1}=-5.11488, \lambda_{2,3}=0.55744 \pm 1.48046 i
$$

Here $\lambda_{1}$ is an negative real number, while $\lambda_{2}$ and $\lambda_{3}$ are a pair of complex conjugate eigenvalues with positive real parts. Thus, the equilibrium $E_{1}$ is a saddle-focus point, which is unstable. For the second equilibrium $E_{2}(-2,-2,0)$, the eigenvalues are identical to those of $E_{1}$. Similarly, for the equilibrium $E_{3,4}$, The eigenvalues are

$$
\lambda_{1}=-4.93056, \lambda_{2,3}=0.465279 \pm 2.75167 i
$$

which also are unstable. 


\section{THE THEORY OF PASSIVE CONTROL}

Consider the following differential:

$$
\begin{aligned}
& \dot{u}=\Lambda(u)+\Upsilon(u) \Theta, \\
& v=\Delta(u),
\end{aligned}
$$

where $u \in \mathbb{R}^{n}$ is state variable, $\Lambda(u)$ and $\Upsilon(u)$ are the smooth vector fields, $\Theta \in \mathbb{R}^{m}$ is the control function, $n>m$ and $\Delta(u)$ is a smooth mapping.

Definition 1 [26, 43] If the matrix $L_{\Upsilon} \Delta(0)=\frac{\partial \Delta}{\partial u} \Upsilon(u)$ is nonsingular, system (11) is said to have relative degree $[1,1, \ldots, 1]$ at $u=0$.

Definition 2 [26, 43] System (11) is said to be $C^{\mathrm{r}}$-passive if there exists a $C^{\mathrm{r}}$-positive real valued function $V(u), V(0)=0$, called storage function, such that $\forall t \geq 0$, the following dissipation inequality holds:

$$
V(u)-V(0) \leq \int_{0}^{t} \Theta^{T}(s) v(s) d s
$$

The parametric version of the normal form of system (11) can be written as follows:

$$
\begin{aligned}
& \dot{\zeta}=\phi_{0}(\zeta)+\chi(\zeta, v) v, \\
& \dot{v}=\varphi(\zeta, v)+\mu(\zeta, v) \Theta
\end{aligned}
$$

where a new coordinate of the system $(13)$ is $(\zeta, v)$, locally defined in the neighborhood of the origin, $\zeta \in \mathbb{R}^{n-\mathrm{m}}$ and $\mu(\zeta, v)$ is nonsingular for all $(\zeta, v)$ in the neighborhood of the origin [26, 43]. Remark 1 Setting $v=0$ in system (13), yields the zero dynamic system:

$$
\dot{\zeta}=\phi_{0}(\zeta)
$$

where the stability of zero dynamics is a necessary condition for passivity control design.

Definition 3 [26, 43] Suppose $L_{\Upsilon} \Delta(0)$ is nonsingular, then system (11) is said to be minimum phase if its zero dynamics is asymptotically stable. In other words, there exists the function $W(\zeta)$ (called Lyapunov function of $\left.\phi_{0}(\zeta)\right)$ which is positive-definite and differentiable in $\zeta$ such that:

$$
\frac{\partial W(\zeta)}{\partial \zeta} \phi_{0}(\zeta) \leq 0
$$

$\forall \zeta$ in a neighborhood of $\zeta=0$.

Theorem 1 [26, 44] If the system (11) is a minimum phase system, the system (12) will be equivalent to a passive system and asymptotically stabilized at an equilibrium point if we let the local feedback control as follows:

$$
\Theta=\mu^{-1}(\zeta, v)\left(-\varphi(\zeta, v)-\left(\frac{\partial W(\zeta)}{\partial \zeta} \chi(\zeta, v)\right)^{T}-\gamma v+\beta\right)
$$

where $\gamma$ is a positive real value and $\beta$ is an external signal vector that is connected with the reference input. System (17) after control as shown in Figure 3. 

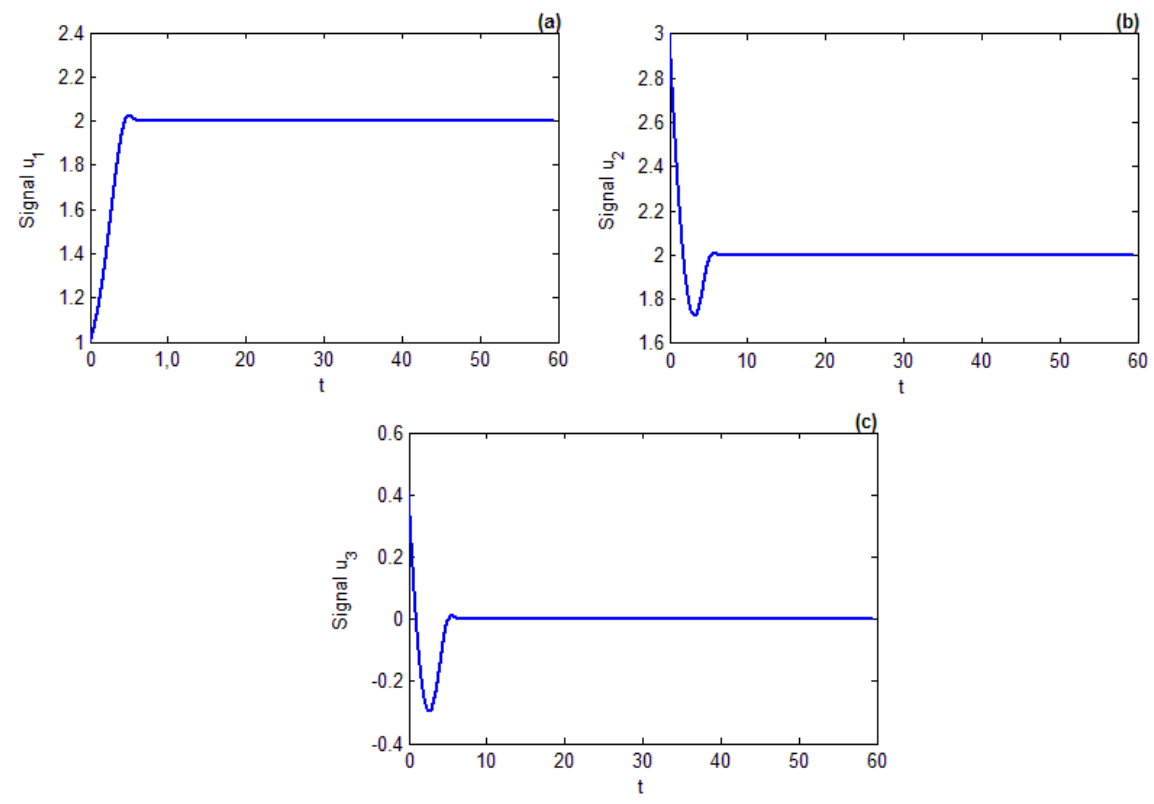

Figure 3. System (17) after control

\section{THE CONTROL OF SYSTEM (1) USING PASSIVE CONTROL} be written as:

To control system (1), we add the control function to the first equation. So, the controller system can

$$
\begin{aligned}
& \dot{u}_{1}=u_{2} u_{3}+\Theta, \\
& \dot{u}_{2}=u_{1}\left|u_{1}\right|-u_{2}\left|u_{2}\right|, \\
& \dot{u}_{3}=a\left|u_{1}\right|-b u_{2}^{2} .
\end{aligned}
$$

The main goal is to design the appreciate controller function $\Theta$ to stabilize system (17).

Theorem 2 If we choose the controller as follows

$$
\Theta=-u_{2} u_{3}-u_{2}\left|u_{1}\right|-\gamma u_{1}+\beta
$$

where $\gamma$ is a positive real constant, then the chaotic system (17) will be asymptotically stabilized at the fixed point.

Proof. Clearly, $L_{\Upsilon} \Delta(0)=1$, where

$$
\Delta=u_{1}, \Upsilon=[1,0,0]^{T},
$$

so according to definition 1 , system (17) have relative degree $[1,1, \ldots, 1]$. Let $\zeta_{1}=u_{2}, \zeta_{2}=u_{3}, v=u_{1}$, the (17) can be rewritten as:

$$
\begin{gathered}
\dot{\zeta}_{1}=v|v|-\zeta_{1}\left|\zeta_{1}\right| \\
\dot{\zeta}_{2}=a|v|-b \zeta_{1}^{2} \\
\dot{v}=\zeta_{1} \zeta_{2}+\Theta .
\end{gathered}
$$

Comparing (20) by (13) one has

$$
\begin{aligned}
\phi_{0}(\zeta) & =\left[\begin{array}{ll}
-\zeta_{1}\left|\zeta_{1}\right| & -b \zeta_{1}^{2}
\end{array}\right]^{T}, \\
\chi(\zeta, v) & =\left[\begin{array}{ll}
|v| & a \operatorname{sign}(v)
\end{array}\right]^{T}, \\
\varphi(\zeta, v) & =\zeta_{1} \zeta_{2} \\
\mu(\zeta, v) & =1 .
\end{aligned}
$$


Let

$$
W(\zeta)=\frac{1}{2} \zeta_{1}^{2},
$$

with $W(0)=0$, then we have

$$
\begin{aligned}
\dot{W}(\zeta) & =\frac{\partial W(\zeta)}{\partial \zeta} \dot{\zeta}=\frac{\partial W(\zeta)}{\partial \zeta} \phi_{0}(\zeta) \\
= & {\left[\begin{array}{ll}
\zeta_{1} & 0
\end{array}\right]\left[\begin{array}{ll}
-\zeta_{1}\left|\zeta_{1}\right| & -b \zeta_{1}^{2}
\end{array}\right]^{T} } \\
= & -\zeta_{1}^{2}\left|\zeta_{1}\right| \leq 0 .
\end{aligned}
$$

Regarding to definition 3, system (17) is minimum phase system. Consequently, based on theorem 1 , one can design the controller as

$$
\Theta=-\zeta_{1} \zeta_{2}-\zeta_{1}|v|-\gamma v+\beta,
$$

Namely

$$
\Theta=-u_{2} u_{3}-u_{2}\left|u_{1}\right|-\gamma u_{1}+\beta .
$$

Remark [26] the attractors of the new chaotic system (17) after control are converted to non-trivial equilibrium $\mathrm{E}_{1}$ point if $\beta=2 \gamma+2|2|$. For the numerical simulation, the fourth-order Runge-Kutta method is used to solve the system of differential (17), with step size equal 0.001 in numerical simulations. By taking $\gamma$ equals to 0.2 and the initial conditions of $(17)$ are $\mathrm{u}_{1}(0)=1, \mathrm{u}_{2}(0)=3, \mathrm{u}_{3}=0.4$. As expected, one can observe that the trajectories of the new chaotic system (17) asymptotically stabilized at equilibrium point $E_{1}$ as illustrated in Figure 3.

\section{CIRCUIT DESIGN OF THE NEW CHAOTIC SYSTEM}

Chaos phenomenon is widely applied in the field of engineering. Specifically, electronic circuits [44-50], secure communication [51], robotic [52], random bits generator [53], and voice encryption [54]. In this section, we describe a possible circuit to implement new chaotic system with line of equilibria (1) as presented in Figure 4. The circuit consists of twenty-one resistors, three capacitors, three integrators (U1A-U3A), three inverting amplifiers (U4A-U6A), four operational amplifiers (U7A-U10A) for absolute nonlinearity, which are implemented with the operational amplifier TL082CD. The circuit has two diodes (D1 (1N4148), D2(1N4148), which provide the signal $|\mathrm{Y}|$, two diodes (D3 (1N4148), D4(1N4148) which produce the signal $|\mathrm{X}|$ and four multipliers (AD633JN).

In this study, a linear scaling is considered as follows:

$$
\left\{\begin{array}{l}
\dot{x}=2 y z \\
\dot{y}=2 x|x|-2 y|y| \\
\dot{z}=a|x|-2 b y^{2}
\end{array}\right.
$$

By applying Kirchhoff's circuit laws, the corresponding circuital equations of the designed circuit can be written as

$$
\left\{\begin{array}{l}
\dot{x}=\frac{1}{C_{1} R_{1}} y z \\
\dot{y}=\frac{1}{C_{2} R_{2}} x|x|-\frac{1}{C_{2} R_{3}} y|y| \\
\dot{z}=\frac{1}{C_{3} R_{4}}|x|-\frac{1}{C_{3} R_{5}} y^{2}
\end{array}\right.
$$

We choose the values of the circuital elements as: $R_{1}=R_{2}=R_{3}=20 \mathrm{k} \Omega, R_{4}=R_{5}=25 \mathrm{k} \Omega, R_{6}=R_{7}=R_{8}=R_{9}=$ $R_{10}=R_{11}=R_{12}=R_{13}=R_{14}=R_{15}=R_{16}=R_{17}=R_{18}=R_{19}=R_{20}=R_{21}=10 \mathrm{k} \Omega, C_{1}=C_{2}=C_{3}=10 \mathrm{nF}$ 
In system (27), the variables $x, y$ and $z$ correspond to the voltages in the outputs of the integrators U1A-U3A. The supplies of all active devices are \pm 15 volt. The MultiSIM projections of chaotic attractors with line equilibria are described in Figures 5 (a-c). The numerical simulations with MATLAB see Figure 1 are similar with the circuital ones see Figure 5.

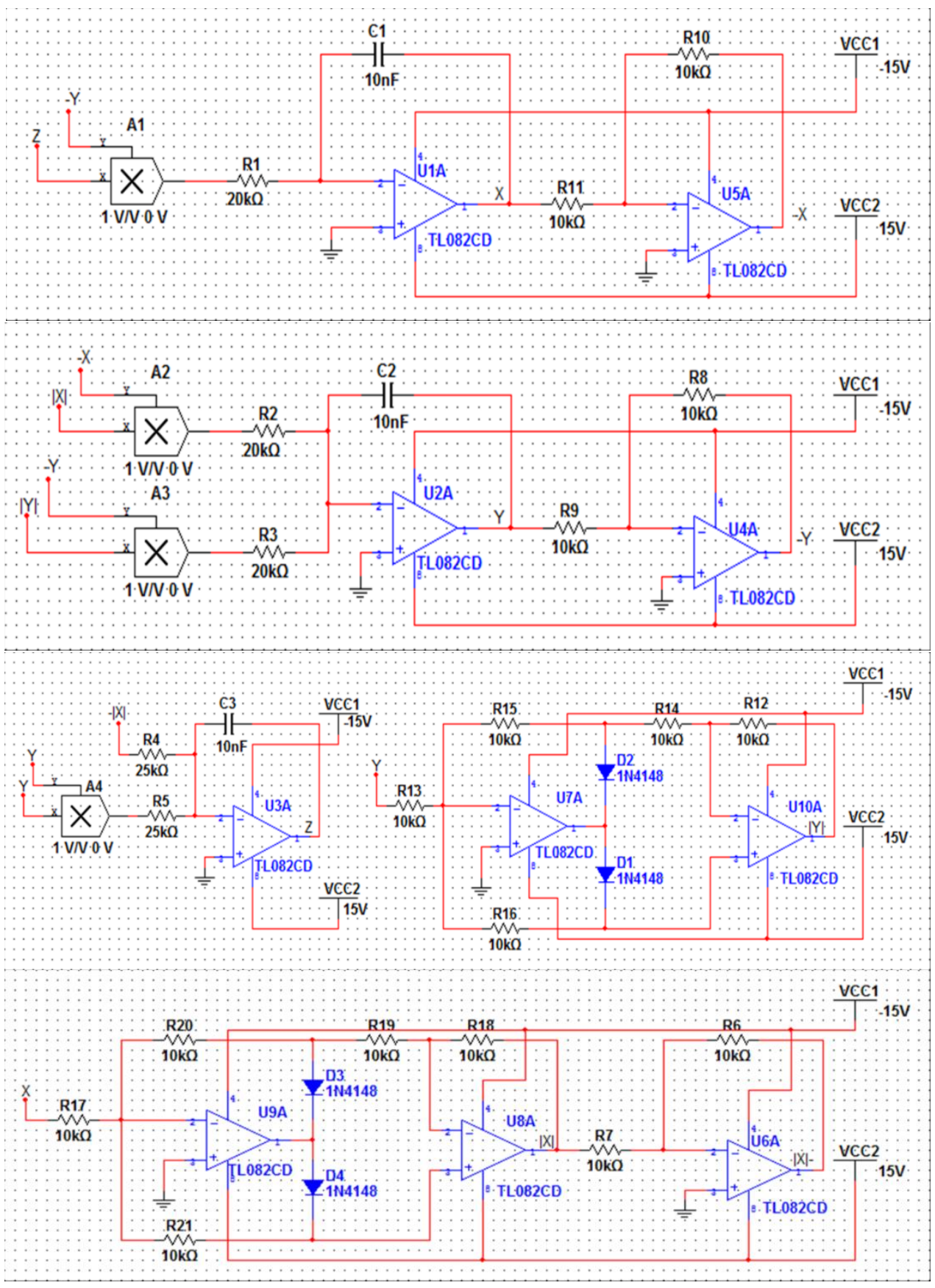

Figure 4. Schematic of the proposed new chaotic system by using MultiSIM 


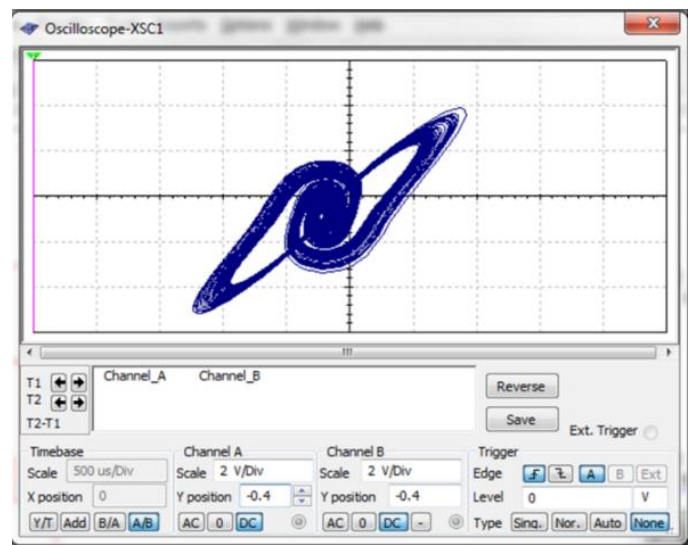

(a)

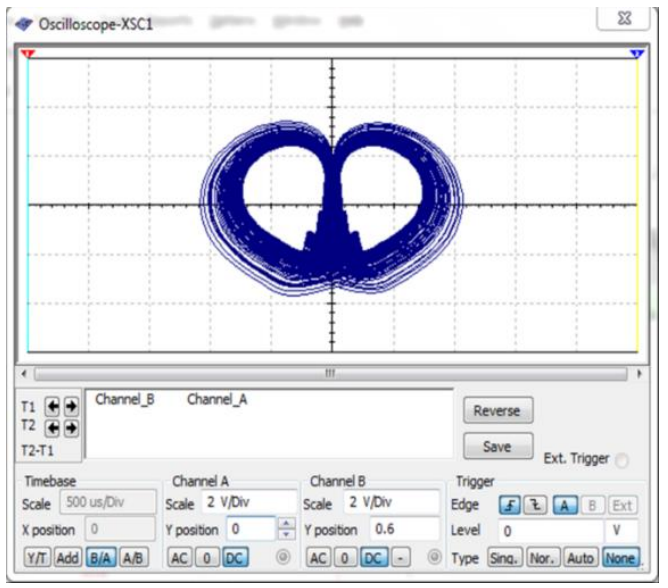

(b)

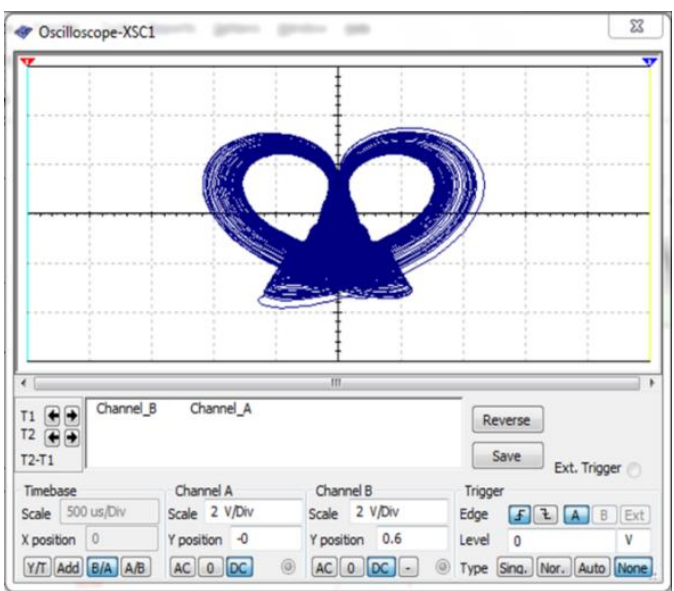

(c)

Figures 5. The phase portraits of new chaotic system (1) observed on the oscilloscope in different planes (a) $\mathrm{x}-\mathrm{y}$ plane, (b) y-z plane and (c) x-z plane by MultiSIM

\section{CONCLUSION}

A new chaotic system with line of equilibria has been investigated. The proposed new chaotic system has rich dynamics as confirmed by eigenvalue structure, chaotic attractors, Lyapunov exponents, bifurcation diagram and Poincaré map. In addition, the possibility of passive control of a new chaotic system with line of equilibria has been analyzed and confirmed. Moreover, electronic circuit has been implemented and tested using the MultiSIM software. Comparison of the oscilloscope output and numerical simulations using MATLAB, showed good qualitative agreement between the chaotic system and circuit design. Further analyses like engineering application on robotic, random bits generator and secure communication system are interesting issues for future work.

\section{ACKNOWLEDGEMENTS}

The authors thank the Government of Malaysia for funding this research under the Fundamental Research Grant Scheme (FRGS/1/2018/ICT03/UNISZA/02/2) and also Universiti Sultan Zainal Abidin, Terengganu, Malaysia.

\section{REFERENCES}

[1] G. A. Leonov, et al., "Hidden Oscillations in Dynamical Systems," Trans. Syst. Contr, 6, 54-67, 2011.

[2] G. A. Leonov, et al., "Hidden Attractor in Smooth Chua Systems," Physica D: Nonlinear Phenomena, vol. 241, 1482-1486, 2012.

[3] E. N. Lorenz, "Deterministic Nonperiodic Flow," Journal of the atmospheric sciences, vol. 20, 130-141, 1963.

[4] O. E. Rössler, "An Equation for Continuous Chaos,” Physics Letters A, vol. 57, 397-398, 1976. 
[5] J. Lü and G. Chen, "A New Chaotic Attractor Coined," International Journal of Bifurcation and chaos, vol. 12, pp. 659-661, 2002.

[6] G. Chen and T. Ueta, "Yet Another Chaotic Attractor," International Journal of Bifurcation and chaos, vol. 9, pp. 1465-1466, 1999.

[7] A. M. Rucklidge, "Chaos In Models of Double Convection," Journal of Fluid Mechanics, vol. 237, pp. 209-229, 1992.

[8] J. C. Sprott, “A New Class of Chaotic Circuit,” Physics Letters A, vol. 266, 19-23, 2000.

[9] S. Jafari, et al., "Elementary Quadratic Chaotic Flows with no Equilibria," Physics Letters A, vol. 377, pp. 699-702, 2013.

[10] X. Wang and G. Chen, "A Chaotic System with Only One Stable Equilibrium," Communications in Nonlinear Science and Numerical Simulation, vol. 17, pp. 1264-1272, 2012.

[11] P. Zhou and F. Yang, "Hyperchaos, Chaos, and Horseshoe in a 4D Nonlinear System with an Infinite Number of Equilibrium Points," Nonlinear Dynamics, vol. 76, pp. 473-480, 2014.

[12] N. V. Kuznetsov, "Hidden Attractors in Fundamental Problems And Engineering Models: A Short Survey," In AETA 2015: Recent Advances in Electrical Engineering and Related Science, pp. 13-25, 2015.

[13] B. R. Andrievsky, et al., "Hidden Oscillations in Aircraft Flight Control System With Input Saturation," 5th IFAC Workshop on Periodic Control Systems, pp. 75-79, 2013.

[14] G. A. Leonov, et al., "Hidden Oscillations in Mathematical Model Of Drilling System Actuated By Induction Motor With A Wound Rotor," Nonlinear Dynamics, vol. 77, 277-288, 2014.

[15] G. A. Leonov, et al., "Hidden Attractor And Homoclinic Orbit In Lorenz-Like System Describing Convective Fluid Motion In Rotating Cavity," Communications in Nonlinear Science and Numerical Simulation, vol. 28, pp. 166-174, 2015.

[16] Z. T. Zhusubaliyev and E. Mosekilde, "Multistability and Hidden Attractors In A Multilevel Dc/Dc Converter," Mathematics and Computers in Simulation, vol. 109, pp. 32-45, 2015.

[17] S. Jafari and J. C. Sprott, "Simple chaotic flows with a line equilibrium," Chaos, Solitons \& Fractals, vol. 57, pp. 79-84, 2013.

[18] C. Li and J. C. Sprott, "Chaotic Flows with A Single Nonquadratic Term," Physics Letters A, vol. 378, pp. 178-183, 2014.

[19] C. Li, et al., "Constructing Chaotic Systems with Total Amplitude Control," International Journal of Bifurcation and Chaos, vol. 25, 1530025, 2015.

[20] C. Li, et al., "Bistability In A Hyperchaotic System With a Line Equilibrium," Journal of Experimental and Theoretical Physics, vol. 118, pp. 494-500, 2014.

[21] Q. Li, et al., "Hyperchaos and Horseshoe In A 4d Memristive System With A Line Of Equilibria And Its Implementation". International Journal of Circuit Theory and Applications, vol. 42, pp. 1172-1188, 2014.

[22] J. P. Singh and B. K. Roy, "The Simplest 4-D Chaotic System with Line Of Equilibria, Chaotic 2-Torus and 3-Torus Behaviour," Nonlinear Dynamics, vol. 89, pp. 1-18, 2017.

[23] S. Vaidyanathan, "A Novel 3-D Jerk Chaotic System with Three Quadratic Nonlinearities And Its Adaptive Control," Archives of Control Sciences, vol. 26, pp. 19-47, 2016.

[24] S. Vaidyanathan and S. Sampath, "Anti-Synchronization Of Four-Wing Chaotic Systems Via Sliding Mode Control," International Journal of Automation and Computing, vol. 9, pp. 274-279, 2012.

[25] N. Bigdeli and H. A. Ziazi, "Design of Fractional Robust Adaptive Intelligent Controller For Uncertain Fractional-Order Chaotic Systems Based On Active Control Technique," Nonlinear Dynamics, vol. 87, pp. 1703-1719, 2017.

[26] G. M. Mahmoud, et al., "Passive Control of n-Dimensional Chaotic Complex Nonlinear Systems," Journal of Vibration and Control, vol. 19, pp. 1061-1071, 2013.

[27] L. M. Pecora and T. L. Carroll," Synchronization in Chaotic Systems," Physical Review Letters, vol. 64, pp. 821-825, 1990.

[28] X. Li and S. Song, "Stabilization of Delay Systems: Delay-Dependent Impulsive Control," IEEE Transactions on Automatic Control, vol. 62, pp. 406-411, 2017.

[29] Y. Li, et al., "Adaptive Fuzzy Control Design for Stochastic Nonlinear Switched Systems With Arbitrary Switchings And Unmodeled Dynamics," IEEE Transactions On Cybernetics, vol. 47, pp. 403-414, 2017.

[30] Q. Wei, et al., "Off-Policy Irl Optimal Tracking Control for Continuous-Time Chaotic Systems," Studies in Systems, Decision and Control, vol. 103, pp. 201-214, 2018.

[31] T. H. Chien and Y. C. Chen, "An On-Line Tracker for a Stochastic Chaotic System Using Observer/Kalman Filter Identification Combined with Digital Redesign Method," Algorithms, vol. 10, pp. 25-36, 2017.

[32] G. M. Mahmoud, et al., "Chaos Control of Integer and Fractional Orders of Chaotic Burke-Shaw System Using Time Delayed Feedback Control," Chaos, Solitons \& Fractals, vol. 104, pp. 680-692, 2017.

[33] G. M. Mahmoud, et al., "Controlling Hyperchaotic Complex Systems with Unknown Parameters Based On Adaptive Passive Method," Chinese Physics B, vol. 22, 060508, 2013.

[34] W. Li, et al., "Stable Kinematic Teleoperation Of Wheeled Mobile Robots With Slippage Using Time-Domain Passivity Control," Mechatronics, vol. 39, pp. 196-203, 2016.

[35] L. Xie, "Passivity Analysis and Passification For Uncertain Signal Processing Systems," IEEE Transactions on Signal Processing, vol. 46, pp. 2394-2403, 1998.

[36] Q. Dong-lian, et al., "Passive Control f Permanent Magnet Synchronous Motor Chaotic Systems," Journal of Zhejiang University-Science A, vol. 6, pp. 728-732, 2005.

Int J Elec \& Comp Eng, Vol. 9, No. 4, August 2019 : 2365 - 2376 
[37] D. H. Ji, et al., Passivity-Based Control for Hopfield Neural Networks Using Convex Representation, Applied Mathematics and Computation, vol. 217, pp. 6168-6175, 2011.

[38] K. Kemih, "Control of Nuclear Spin Generator System Based On Passive Control," Chaos, Solitons \& Fractals, vol. 41, pp. 1897-1901, 2009.

[39] W. Xiang-Jun, et al., "Chaos Synchronization of Rikitake Chaotic Attractor Using The Passive Control Technique," Nonlinear Dynamics, vol. 53, pp. 45-53, 2008.

[40] A. Goswami, et al.,"A Study of The Passive Gait Of A Compass-Like Biped Robot: Symmetry and Chaos," The International Journal of Robotics Research, vol. 17, pp. 1282-1301, 1998.

[41] G. M. Mahmoud, et al., "On Projective Synchronization of Hyperchaotic Complex Nonlinear Systems Based On Passive Theory For Secure Communications,” Physica Scripta, vol. 87, 055002, 2013.

[42] A.Wolf, et al., "Determining Lyapunov Exponents From a Time Series," Physica D, vol. 16, pp. 285-317, 1985.

[43] C. I. Byrnes, et al., "Passivity, Feedback Equivalence, and the Global Stabilization of Minimum Phase Nonlinear Systems," IEEE Transactions on automatic control, vol. 36, pp. 1228-1240, 1991.

[44] C. Li, et al., "Linear Synchronization And Circuit Implementation Of Chaotic System With Complete Amplitude Control," Chinese Physics B, vol. 26, 120501, 2017.

[45] C. Li, et al., "A New Piecewise Linear Hyperchaotic Circuit," IEEE Transactions on Circuits and Systems II: Express Briefs, vol. 61, pp. 977-981, 2014.

[46] C. Li, et al., "A New Chaotic Oscillator with Free Control," Chaos: An Interdisciplinary Journal of Nonlinear Science, vol. 27, 083101, 2017

[47] A. Sambas, et al., "A New Chaotic System with a Pear-Shaped Equilibrium and its Circuit Simulation," International Journal of Electrical and Computer Engineering, vol. 8, no. 6, pp. 4951-4958, 2018.

[48] S. Vaidyanathan, et al., "A New Chaotic System with Axe-Shaped Equilibrium, Its Circuit Implementation and Adaptive Synchronization," Archives of Control Sciences, vol. 28, pp. 443-462, 2018.

[49] S. Mobayen, et al., "A Novel Chaotic System with Boomerang-Shaped Equilibrium, Its Circuit Implementation and Application to Sound Encryption," Iranian Journal of Science and Technology, Transactions of Electrical Engineering, vol. 43, pp. 1-12, 2019.

[50] S. Vaidyanathan, et al., "A New Three-Dimensional Chaotic System with a Cloud-Shaped Curve of Equilibrium Points, Its Circuit Implementation and Sound Encryption,” International Journal of Modelling, Identification and Control, vol. 30, pp. 184-196, 2018.

[51] A. Sambas, et al., "Mathematical Modelling of Chaotic Jerk Circuit and Its Application in Secure Communication System," Studies in Fuzziness and Soft Computing, vol. 337, pp. 133-153, 2016.

[52] S. Vaidyanathan, et al., "A New Three-Dimensional Chaotic System With A Hidden Attractor, Circuit Design And Application In Wireless Mobile Robot," Archives of Control Sciences, vol. 27, pp. 541-554, 2017.

[53] B. Karakaya, et al., "Chaotic Cellular Neural Network-Based True Random Number Generator," International Journal of Circuit Theory and Applications, vol. 45, pp. 1885-1897, 2017.

[54] S. Vaidyanathan, et al., "Analysis, Synchronisation and Circuit Implementation of a Novel Jerk Chaotic System and its Application for Voice Encryption," International Journal of Modelling, Identification and Control, vol. 28, pp. 153-166, 2017.

\section{BIOGRAPHIES OF AUTHORS}

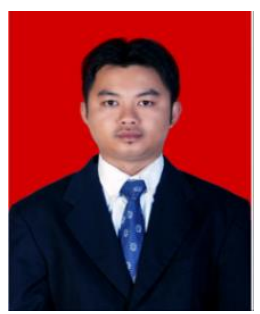

Aceng Sambas is currently a Lecturer at the Muhammadiyah University of Tasikmalaya, Indonesia since 2015. He received his M.Sc in Mathematics from the Universiti Sultan Zainal Abidin (UniSZA), Malaysia in 2015. His current research focuses on dynamical systems, chaotic signals, electrical engineering, computational science, signal processing, robotics, embedded systems and artificial intelligence.

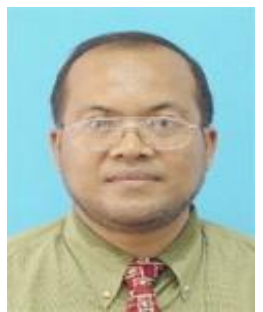

Mustafa Mamat is currently a Professor and the Dean of Graduate School at Universiti Sultan Zainal Abidin (UniSZA), Malaysia since 2013. He was first appointed as a Lecturer at the Universiti Malaysia Terengganu (UMT) in 1999. He obtained his PhD from the UMT in 2007 with specialization in optimization. Later on, he was appointed as a Senior Lecturer in 2008 and then as an Associate Professor in 2010 also at the UMT. To date, he has successfully supervised more than 60 postgraduate students and published more than 150 research papers in various international journals and conferences. His research interests include conjugate gradient methods, steepest descent methods, Broyden's family and quasi-Newton methods. 


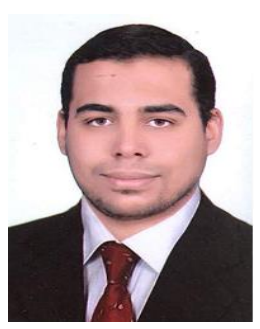

Ayman A. Arafa is currently a Lecturer at Sohag University, Egypt. After earning his Bachelor's degree of Sciences (Major: Mathematics) (excellent with honor degree) (May-2009), he has awarded a Master degree in Mathematics, Dynamical Systems (July-2013), from the Faculty of Science, Sohag University, Sohag, Egypt. He received his $\mathrm{PhD}$ in Mathematics from Sohag University in 2018. He is interested in Dynamical systems, time delay dynamical systems, chaos, bifurcation.

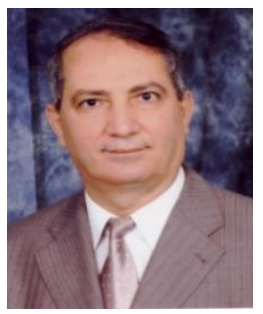

Gamal M. Mahmoud got his Ph. D. degree from Clarkson University, Potsdam, New York 1987. The areas of research and interest are Nonlinear Dynamical Systems (both real and complex systems), Stochastic Systems (both real and complex systems), difference Equations. and Nonlinear Differential Equations. He has joint research with active groups from different universities of USA, Germany, Italy, Greece, Spain, Japan, Egypt and China. He organized and participated many international conferences in several countries. He gave several lectures in different countries, e.g. USA, Japan, Germany, Italy, Greece, China, Egypt, United Arab Emirates, Syria and Saudi Arabia. He published more than 65 papers in I.S.I Journals and 16 papers in Int. Conferences and other journals. He is an editor of many Scientific journals. $($ H-index $=22$, Citations $=1518$ (Scopus, 65 Cited Documents-up to 1-1-2018)

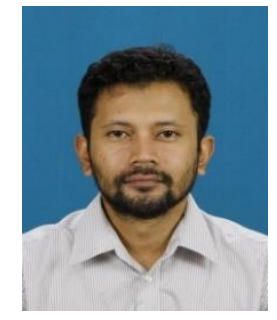

Mohamad Afendee Mohamed received his PhD in Mathematical Cryptography in 2011 and currently serves as an associate professor at Universiti Sultan Zainal Abidin. His research interests include both theoretical and application issues in the domain of data security, and mobile and wireless networking.

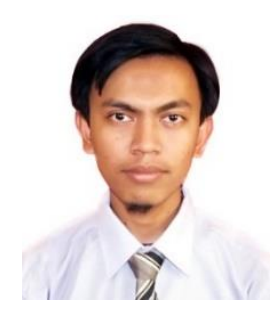

Mada Sanjaya WS received his Ph.D in Mathematics from the University Malaysia Terengganu, Malaysia in 2012. He was first appointed as a Lecturer at the UIN Sunan Gunung Djati Bandung, Indonesia in 2009. His research interests include nonlinear dynamical systems, chaotic systems, artificial intelligence, soft computing and robotic systems. 